PROCEEDINGS OF THE

AMERICAN MATHEMATICAL SOCIETY

Volume 129, Number 4, Pages 1169-1177

S 0002-9939(00)05656-2

Article electronically published on October 19, 2000

\title{
TORUS ACTIONS ON SYMPLECTIC ORBI-SPACES
}

\author{
TANYA SCHMAH
}

(Communicated by Ronald A. Fintushel)

\begin{abstract}
Which $2 n$-dimensional orbi-spaces have effective symplectic $k$ torus actions? As shown by Lerman and Tolman (1997) and Watson (1997), this question reduces to that of characterizing the finite subgroups of centralizers of tori in the real symplectic group $S p(2 n, \mathbb{R})$. We resolve this question, and generalize our method to a calculation of the centralizers of all tori in $S p(2 n, \mathbb{R})$.
\end{abstract}

\section{INTRODUCTION}

This work is motivated by the study of torus actions on symplectic orbifolds by Lerman and Tolman $[\mathrm{LT}]$, and is an extension of work by Watson $[\mathrm{W}$. on circle actions on 4-dimensional orbi-spaces. As discussed in [LT], orbifolds arise in geometric mechanics as reduced phase spaces. The specific question we are concerned with is: for a given $k$, which $2 n$-dimensional orbi-spaces have effective symplectic $T^{k}$ (k-torus) actions? As shown in [LT] and [W], this question reduces to that of characterizing the finite subgroups of centralizers of tori in the real symplectic group $S p(2 n, \mathbb{R})$ (see Lemma 2.1 below). In resolving this question, we were able to generalize our method to a calculation of the centralizers of all tori in $\operatorname{Sp}(2 n, \mathbb{R})$, which may be of interest in its own right.

A symplectic orbi-space is $\mathbb{R}^{2 n} / \Gamma$ for some finite subgroup $\Gamma$ of $S p(2 n, \mathbb{R})$. The group of symplectomorphisms of $\mathbb{R}^{2 n} / \Gamma$, denoted $S p\left(\mathbb{R}^{2 n} / \Gamma\right)$, is defined to be $N(\Gamma) / \Gamma$. A symplectomorphism $\varphi$ of $\mathbb{R}^{2 n} / \Gamma$ acts on $\mathbb{R}^{2 n} / \Gamma$ by $\varphi(\Gamma v)=\Gamma \varphi(v)$. A symplectic action on $\mathbb{R}^{2 n} / \Gamma$ is a $\Gamma$-invariant symplectic action on $\mathbb{R}^{2 n}$.

The main results are Theorems 4.7 and 5.1. It is hoped that the latter, resolving the question of which orbi-spaces have symplectic $T^{k}$ actions, will be useful in a future classification of all orbifolds that admit effective torus actions. Lerman and Tolman have already classified $2 n$-dimensional orbifolds admitting an effective $n$ torus action $[\mathrm{LT}]$. The results here are a generalization of the work of Watson [W], in which he proves Theorem 5.1 for $n=2$ and $k=1$. Both the present paper and [W] make use of results in [LT].

Received by the editors March 23, 1999 and, in revised form, July 7, 1999.

2000 Mathematics Subject Classification. Primary 53D22; Secondary 53D30, 53D20, 70H15, $57 \mathrm{~S} 15$.

Key words and phrases. Symplectic orbifolds, Hamiltonian torus actions, centralizers of tori.

This work originally appeared in a Master's thesis submitted to Bryn Mawr College. The author would like to thank Bryn Mawr College and her advisor Stephanie Frank Singer.

(C)2000 American Mathematical Society 
Throughout this article, all centralizers mentioned will be with respect to $S p(2 n, \mathbb{R})$.

\section{The RELATIONSHIP OF TORUS ACTIONS TO CENTRALIZERS OF TORI}

The following lemma gives the relationship between torus actions on symplectic orbi-spaces and centralizers of tori in $\operatorname{Sp}(2 n, \mathbb{R})$. Most of the result is contained in LT, 3.1, 6.1]; since that version is only concerned with $T^{n}$ actions in $\mathbb{R}^{2 n}$, and the centralizer of $T^{n}$ in $S p(2 n, \mathbb{R})$ is itself, centralizers are not mentioned. A version applicable to all torus actions is given in [W]; it is only stated for $n=2$, but the proof generalizes easily. We present a collected and slightly modified proof here.

Lemma 2.1 ( $[\mathrm{LT},[\mathrm{W}])$. Let $\Gamma$ be a finite subgroup of $S p(2 n, \mathbb{R})$. Then $\mathbb{R}^{2 n} / \Gamma$ admits an effective symplectic $T^{k}$ action if and only if $\Gamma$ is a subgroup of the centralizer $($ in $\operatorname{Sp}(2 n, \mathbb{R})$ ) of some $k$-torus in $\operatorname{Sp}(2 n, \mathbb{R})$.

Proof. $(\Longrightarrow)$ Suppose $\mathbb{R}^{2 n} / \Gamma$ admits an effective symplectic $T^{k}$ action. Then there is some $k$-torus $T$ in $N(\Gamma) / \Gamma$. Let $\hat{T}=\pi^{-1}(T)$ be the preimage of $T$ by the quotient map $N(\Gamma) \rightarrow N(\Gamma) / \Gamma$, and let $\tilde{T}$ be the identity component of $\hat{T}$. We will show that $\tilde{T}$ is a $k$-torus and $\hat{T} \subset Z(\tilde{T})$, which will complete this half of the proof, since $\Gamma \subset \hat{T}$. Since $\tilde{T}$ is a connected component, it is closed, and so it must be a Lie group. To show compactness of $\hat{T}$, and hence $\tilde{T}$, let $\left(q_{i}\right)$ be a sequence in $\hat{T}$. Since the original torus $T$ is compact, $\left(\pi\left(q_{i}\right)\right)$ must have a cluster point, $p$. Since $\Gamma$ is finite, one of the preimages of $p$ must be a cluster point of $\left(q_{i}\right)$. Next we show that $\hat{T} \subset Z(\tilde{T})$. Let $a \in \hat{T}$. Define $f_{a}: \hat{T} \rightarrow \hat{T}$ by $f_{a}(b)=a b a^{-1} b^{-1}$. Since $\pi(\hat{T})$ is abelian, we must have $\pi\left(f_{a}(\hat{T})\right)=\{\Gamma\}$, so $f_{a}(\hat{T}) \subset \Gamma$. Now $\tilde{T}$ is connected by definition, and $f_{a}$ is continuous, so $f_{a}(\tilde{T})$ is connected. But the only connected subgroup of the finite group $\Gamma$ is the trivial one, so $f_{a}(\tilde{T})=\{I\}$. Since this holds for every element $a$ of $\hat{T}$, we have shown that $\hat{T} \subset Z(\tilde{T})$. One useful consequence of this is that $\tilde{T}$ is abelian; since we have already shown that it is a compact and connected Lie group, it must be a torus. Since $\pi$ is onto $T$ and has a finite kernel, $\tilde{T}$ must be $k$-dimensional. Thus $\tilde{T}$ is a $k$-torus and $\Gamma \subset \hat{T} \subset Z(\tilde{T})$, as required.

$(\Longleftarrow)$ Suppose $\Gamma$ is a finite subgroup of $Z(\tilde{T})$ for some $k$-torus $\tilde{T}$ in $\operatorname{Sp}(2 n, \mathbb{R})$. Then $\tilde{T} \subset N(\Gamma)$, so by the "second isomorphism theorem for Lie groups" we have

$$
\tilde{T} \Gamma / \Gamma \cong \tilde{T} /(\Gamma \cap \tilde{T}) .
$$

From this isomorphism we see that $\tilde{T} \Gamma / \Gamma$ is abelian, compact and connected, so it must be a torus; in fact, it must be a $k$-torus, because $\Gamma \cap \tilde{T}$ is finite. Thus we have constructed an effective symplectic $T^{k}$ action on $\mathbb{R}^{2 n} / \Gamma$.

\section{Centralizers of tori: Reduction to a special Case}

We will now show that we need only consider centralizers of certain very simple tori. These results lead fairly easily to a proof of Theorem 5.1. Though the proof of Theorem 5.1 does not require an explicit computation of the centralizers of all tori in $S p(2 n, \mathbb{R})$, we give the result of such a computation in Theorem 4.7

As a first step, it follows from standard results (see for example BtD]), that all tori in $S p(2 n, \mathbb{R})$ are conjugate to one contained in the following diagonal represen- 
tation of $T^{n}$ :

$$
\mathbb{T}^{n}:=\left\{\left(\begin{array}{ccccc}
\cos \theta_{1} & -\sin \theta_{1} & & & 0 \\
\sin \theta_{1} & \cos \theta_{1} & & & \\
& & \ddots & & \cos \theta_{n} \\
& & & -\sin \theta_{n} \\
0 & & & \sin \theta_{n} & \cos \theta_{n}
\end{array}\right): \theta_{1}, \theta_{2}, \ldots, \theta_{n} \in \mathbb{R}\right\} .
$$

Thus Lemma 2.1 implies the following.

Lemma 3.1. Let $\Gamma$ be a finite subgroup of $S p(2 n, \mathbb{R})$. Then $\mathbb{R}^{2 n} / \Gamma$ admits an effective symplectic $T^{k}$ action if and only if $\Gamma$ is conjugate to a subgroup of the centralizer of some $k$-torus in $\mathbb{T}^{n}$.

We now define some notation for tori in $\mathbb{T}^{n}$. First, a few conventions. We will consider the standard tori $T^{n}$ to be subgroups of $\mathbb{C}^{n}$ in the usual way,

$$
T^{n}=\left\{\left(z_{1}, \ldots, z_{n}\right) \in \mathbb{C}^{n}:\left|z_{i}\right|=1 \text { for all } i\right\} .
$$

We denote by exp the map from $\mathbb{R}^{n}$ to $T^{n}$ given by $\mathbf{t} \longmapsto e^{2 \pi i t}$ (component-wise exponentiation). Define the map diag: $\mathbb{C}^{n} \rightarrow \operatorname{Mat}(n, \mathbb{C})$ by

$$
\operatorname{diag}\left(\begin{array}{c}
z_{1} \\
\vdots \\
z_{n}
\end{array}\right)=\left(\begin{array}{ccc}
z_{1} & & 0 \\
& \ddots & \\
0 & & z_{n}
\end{array}\right)
$$

We will identify $\operatorname{Mat}(n, \mathbb{C})$ with its representation in $\operatorname{Mat}(2 n, \mathbb{R})$ induced by

$$
a+b i \longmapsto\left(\begin{array}{cc}
a & -b \\
b & a
\end{array}\right)
$$

All homomorphisms from $T^{k}$ to $T^{n}$ are of the form $\exp (\mathbf{t}) \longmapsto \exp (M \mathbf{t})$ for some $n \times k$ matrix $M$ with integer entries. For all such matrices $M$, define

$$
\begin{aligned}
\varphi_{M}: T^{k} & \rightarrow \mathbb{T}^{n} \\
\exp (\mathbf{t}) & \longmapsto \operatorname{diag}(\exp (M \mathbf{t}))
\end{aligned}
$$

and let $\Phi_{M}=\operatorname{Im}\left(\varphi_{M}\right)$. All tori in $\mathbb{T}^{n}$ are of this form. Note that $\Phi_{M}$ is a $k$-torus if and only if $M$ has rank $k$ (though $\varphi_{M}$ need not be faithful).

Lemma 3.2. If $C \in Z\left(\Phi_{M}\right)$, then either the $i^{\text {th }}$ row of $M$ equals \pm 1 times the $j^{\text {th }}$ row or (considering $C$ as an element of Mat $\left.(2 n, \mathbb{R})\right)$ the $(i, j)^{\text {th }} 2 \times 2$ block of $C$ is zero. If the $i^{\text {th }}$ row equals the $j^{\text {th }}$ row, then the $(i, j)^{\text {th }}$ block is of the form $\left(\begin{array}{cc}a & b \\ -b & a\end{array}\right)$. If the $i^{\text {th }}$ row equals -1 times the $j^{\text {th }}$ row, then the $(i, j)^{\text {th }}$ block is of the form $\left(\begin{array}{cc}a & b \\ b & -a\end{array}\right)$.

Proof. We have $C \varphi_{M}(\mathbf{s})=\varphi_{M}(\mathbf{s}) C$ for all $\mathbf{s}=\left(s_{1}, \ldots, s_{n}\right) \in T^{k}$. For every $1 \leq \ell \leq$ $k$, we can differentiate this equation at the identity, giving

$$
C\left(\left.\frac{\partial \varphi_{M}}{\partial s_{\ell}}\right|_{\mathbf{s}=0}\right)=\left(\left.\frac{\partial \varphi_{M}}{\partial s_{\ell}}\right|_{\mathbf{s}=0}\right) C .
$$


By direct computation we find that, if $M=\left(m_{i j}\right)$ and the $(i, j)^{t h} 2 \times 2$ block of $C$ is $\left(\begin{array}{ll}a & b \\ c & d\end{array}\right)$, we have

$$
m_{j \ell}\left(\begin{array}{cc}
b & -a \\
d & -c
\end{array}\right)=m_{i \ell}\left(\begin{array}{cc}
-c & -d \\
a & b
\end{array}\right)
$$

from which it follows that

$$
m_{i \ell}^{2}\left(\begin{array}{cc}
-c & -d \\
a & b
\end{array}\right)=m_{i \ell} \cdot m_{j \ell}\left(\begin{array}{cc}
b & -a \\
d & -c
\end{array}\right)=m_{j \ell}^{2}\left(\begin{array}{cc}
-c & -d \\
a & b
\end{array}\right) .
$$

Thus if any of $a, b, c$ or $d$ are nonzero, we see that $m_{i \ell}= \pm m_{j \ell}$. Further, the ratio $\frac{m_{i l}}{m_{j l}}$ is independent of $l$. The result follows.

Lemma 3.3. If $M_{1}$ and $M_{2}$ are $n \times k$ matrices with integer entries and $M_{2}=Q M_{1}$, where $Q$ is a permutation matrix, then $Z\left(\Phi_{M_{2}}\right)=Q Z\left(\Phi_{M_{1}}\right) Q^{-1}$.

Proof. Consider first the case where $Q$ is a transposition of two rows, say rows $i$ and $j$. Let $\mathbf{t} \in \mathbb{R}^{k}$. Since exp acts componentwise, $\exp (Q \mathbf{t})=Q \exp \mathbf{t}$. By a simple computation, we see that for any $\mathbf{s} \in \mathbb{C}^{n}$, we have $\operatorname{diag}(Q \mathbf{s})=Q \operatorname{diag}(\mathbf{s}) Q^{-1}$. So

$$
\begin{aligned}
\Phi_{M_{2}} & =\left\{\operatorname{diag}\left(\exp \left(Q M_{1} \mathbf{t}\right): \mathbf{t} \in \mathbb{R}^{k}\right\}\right. \\
& =\left\{Q \operatorname{diag}\left(\exp \left(M_{1} \mathbf{t}\right)\right) Q^{-1}: \mathbf{t} \in \mathbb{R}^{k}\right\} \\
& =Q \Phi_{M_{1}} Q^{-1} .
\end{aligned}
$$

This implies that $Z\left(\Phi_{M_{2}}\right)=Q Z\left(\Phi_{M_{1}}\right) Q^{-1}$. The result for general $Q$ follows.

Definition 3.4. A matrix $M$ is in $P M$-block form (the "PM" stands for "plus-orminus") if it can be divided horizontally into blocks,

$$
M=\left(\begin{array}{c}
\text { Block } 1 \\
\text { Block } 2 \\
\vdots \\
\text { Block } r
\end{array}\right)
$$

satisfying the following conditions.

- Each block can be subdivided horizontally into two sub-blocks, $\left(\begin{array}{c}A \\ B\end{array}\right)$ called the top half and the bottom half, such that $A$ is nonempty, all rows in $A$ are equal, and if $B$ is nonempty, then all rows in $B$ are equal and each row in $B$ equals -1 times each row in $A$.

- No row is equal to \pm 1 times a row from a different block.

- If there is a zero block, it is the bottom one (Block $r$ ).

Each block satisfying these conditions will be called a PM-block.

Definition 3.5. For any sets of matrices $S_{1} \subset \operatorname{Mat}\left(n_{1}, \mathbb{C}\right)$ and $S_{2} \subset \operatorname{Mat}\left(n_{2}, \mathbb{C}\right)$, define

$$
S_{1} \times S_{\iota}=\left\{\left(\begin{array}{cc}
A_{1} & 0 \\
0 & A_{2}
\end{array}\right): A_{1} \in S_{1} \text { and } A_{2} \in S_{2}\right\} .
$$

Note that if $M=\left(\begin{array}{c}M_{1} \\ \vdots \\ M_{r}\end{array}\right)$, then $\Phi_{M}=\Phi_{M_{1}} \times_{\iota} \ldots \times_{\iota} \Phi_{M_{r}}$. 
Proposition 3.6. If $M$ has integer entries and is in PM-block form, with PMblocks $M_{1}, \ldots M_{r}$, then $Z\left(\Phi_{M}\right)=Z\left(\Phi_{M_{1}}\right) \times_{\iota} \ldots \times_{\iota} Z\left(\Phi_{M_{r}}\right)$.

Proof. Suppose $C \in Z\left(\Phi_{M}\right)$. For every $i$ and $j$, if the $i^{t h}$ and $j^{\text {th }}$ rows of $M$ are in different PM-blocks, then by Lemma 3.2, the $(i, j)^{t h} 2 \times 2$ block of $C$ is zero. So $C$ is of the form

$$
C=\left(\begin{array}{ccc}
C_{1} & & 0 \\
& \ddots & \\
0 & & C_{r}
\end{array}\right)
$$

for some $2 n_{s} \times 2 n_{s}$ matrices $C_{s}$, where $n_{1}, \ldots, n_{r}$ are the sizes of the blocks $M_{1}, \ldots, M_{r}$ respectively.

For matrices of this form, it is clear that $C$ is symplectic if and only if $C_{1}, \ldots, C_{r}$ are. Further, since $\Phi_{M}=\Phi_{M_{1}} \times_{\iota} \ldots \times_{\iota} \Phi_{M_{r}}$, we see that $C \in Z\left(\Phi_{M}\right)$ if and only if each $C_{s} \in Z\left(\Phi_{M_{s}}\right)$.

Note that for every $n \times n$ matrix $M$, there exists some row-permutation matrix $Q$ such that $Q M$ is in PM-block form. Thus Lemma 3.3 and the preceding proposition reduce our problem to one of finding the centralizers of tori $\Phi_{M}$ for matrices $M$ consisting of only one PM-block.

In fact, the results in this section suffice in order to prove our main result, Theorem 5.1 about torus actions on orbi-spaces. However, we are now in a position to calculate the centralizers of all tori in $S p(2 n, \mathbb{R})$.

\section{Computation of the Centralizers of tori}

We now find the centralizers of all tori $\Phi_{M}$ such that $M$ consists of only one PM-block. In general, matrices in the centralizer of $\Phi_{M}$ won't be in $G L(n, \mathbb{C})$. However, we will find conjugates that are. We need to define some new families of matrices.

Definition 4.1. For any $p \leq n$ and $q=n-p$, let $F_{p, q} \in G L(2 n, \mathbb{R})$ be the matrix in which the upper left hand block is the $2 p \times 2 p$ identity matrix, the remainder of the diagonal consists of alternating 1's and -1 's, and the rest of the matrix is zero.

Note that $F_{p, q}=F_{p, q}^{T}=F_{p, q}^{-1}$.

Lemma 4.2. Let $M$ be the $n \times 1$ matrix $(m, \ldots, m,-m, \ldots,-m)^{T}$ for some non-zero integer $m$, where there are $p$ entries of $m$ and $q$ entries of $-m$. Then $F_{p, q} Z\left(\Phi_{M}\right) F_{p, q}^{-1}$ $\subset G L(n, \mathbb{C})$.

Proof. Let $C \in Z\left(\Phi_{M}\right)$, and let $1 \leq i, j \leq n$. If $i$ and $j$ are either both less than or equal to $p$, or both greater than $p$, then Lemma 3.2 shows that the $(i, j)^{\text {th }} 2 \times 2$ block of $C$ is of the form $\left(\begin{array}{cc}a & b \\ -b & a\end{array}\right)$. Direct calculation shows that the $(i, j)^{\text {th }}$ $2 \times 2$ block of $F_{p, q} C F_{p, q}^{-1}$ is either

$$
\left(\begin{array}{cc}
a & b \\
-b & a
\end{array}\right) \quad(\text { if } i, j \leq p), \text { or }\left(\begin{array}{cc}
a & -b \\
b & a
\end{array}\right)(\text { if } i, j>p)
$$


If $i \leq p<j$ or $j \leq p<i$, then Lemma 3.2 shows that $(i, j)^{\text {th }} 2 \times 2$ block of $C$ is of the form $\left(\begin{array}{cc}a & b \\ b & -a\end{array}\right)$. So the $(i, j)^{\text {th }} 2 \times 2$ block of $F_{p, q} C F_{p, q}^{-1}$ is either

$$
\left(\begin{array}{cc}
a & b \\
-b & a
\end{array}\right) \text { (if } i \leq p \text { and } j>p \text { ), or }\left(\begin{array}{cc}
a & -b \\
b & a
\end{array}\right) \text { (if } j \leq p \text { and } i>p \text { ). }
$$

Definition 4.3. For any $p \leq n$ and $q=n-p$, let $I_{p, q}$ be the complex $n \times n$ matrix

$$
\left(\begin{array}{cc}
I_{p} & 0 \\
0 & -I_{q}
\end{array}\right)
$$

where $I_{p}$ is the $p \times p$ identity matrix.

Note that $-i I_{n, 0}$, when regarded as an element of $G L(2 n, \mathbb{R})$, is the matrix $J_{n}$ used to define the symplectic form.

More generally, $F_{p, q} J_{n} F_{p, q}^{-1}=-i I_{p, q}$.

Definition 4.4. Let $U(p, q)=\left\{A \in G L(n, \mathbb{C}) \mid A^{*} I_{p, q} A=I_{p, q}\right\}$. The matrices in these groups are called pseudounitary.

Note that $U(n, 0)=U(0, n)=U(n)$, the unitary group.

Lemma 4.5. The pseudounitary group $U(p, q)=G L(n, \mathbb{C}) \cap F_{p, q} S p(2 n, \mathbb{R}) F_{p, q}^{-1}$.

Note that this is a generalization of the standard result that $U(n)=G L(n, \mathbb{C}) \cap$ $S p(2 n, \mathbb{R})[\mathrm{MS}, 2.17]$.

Proof. Let $A \in G L(n, \mathbb{C})$. In the second line of the following computation we use the fact that $A^{*}$, when $A$ is considered as an element of $G L(n, \mathbb{C})$, corresponds to $A^{T}$ when $A$ is considered as an element of $G L(2 n, \mathbb{R})$. We have

$$
\begin{aligned}
A \in U(p, q) & \Longleftrightarrow A^{*} I_{p, q} A=I_{p, q} \\
& \Longleftrightarrow A^{T} I_{p, q} A=I_{p, q} \\
& \Longleftrightarrow A^{T}\left(-i I_{p, q}\right) A=\left(-i I_{p, q}\right) \\
& \Longleftrightarrow A^{T} F_{p, q} J_{n} F_{p, q}^{-1} A=F_{p, q} J_{n} F_{p, q}^{-1} \\
& \Longleftrightarrow F_{p, q}^{-1} A^{T} F_{p, q} J_{n} F_{p, q}^{-1} A F_{p, q}=J_{n} \\
& \Longleftrightarrow\left(F_{p, q}^{-1} A F_{p, q}\right)^{T} J_{n}\left(F_{p, q}^{-1} A F_{p, q}\right)=J_{n} \\
& \Longleftrightarrow F_{p, q}^{-1} A F_{p, q} \in S p(2 n, \mathbb{R}) \\
& \Longleftrightarrow A \in F_{p, q} S p(2 n, \mathbb{R}) F_{p, q}^{-1} .
\end{aligned}
$$

Definition 4.6. For every $p$ and $q$, let $W(p, q)=F_{p, q}^{-1} U(p, q) F_{p, q}$.

Note that $W(n, 0)=W(0, n)=U(n)$.

We are now able to state the main result of this section.

Theorem 4.7. Let $M$ be an $n \times k$ matrix, with integer entries, in PM-block form. Let $r$ be the number of PM-blocks in $M$, and let $n_{i}$ be the number of rows in the $i^{\text {th }}$ PM-block, $p_{i}$ the number of rows in the top half and $q_{i}=n_{i}-p_{i}$ the number of rows in the bottom half. If all rows of $M$ are nonzero, then

$$
Z\left(\Phi_{M}\right)=W\left(p_{1}, q_{1}\right) \times_{\iota} \cdots \times_{\iota} W\left(p_{r}, q_{r}\right)
$$


and if $M$ does contain some zero rows, then

$$
Z\left(\Phi_{M}\right)=W\left(p_{1}, q_{1}\right) \times_{\iota} \cdots \times_{\iota} W\left(p_{r-1}, q_{r-1}\right) \times_{\iota} S p\left(2 n_{r}, \mathbb{R}\right) .
$$

The proof will follow easily from the following result.

Proposition 4.8. Suppose $M$ is a nonzero $n \times k$ matrix, with integer entries, consisting of only one PM-block, with $p$ rows in the top half and $q$ rows in the bottom half. Then $Z\left(\Phi_{M}\right)=W(p, q)$. (This was proved for $n=2$ and $k=1$ in W.)

Proof. The rank of $M$ is 1 , so $\Phi_{M}$ is also the image of a faithful homomorphism from $T^{1}$ to $S p(2 n, \mathbb{R})$. So without loss of generality, we will assume that $M$ has only one column, in other words, that $M=(m, \ldots, m,-m, \ldots,-m)^{T}$ for some nonzero integer $m$. We can now easily prove that $Z\left(\Phi_{M}\right) \subset W(p, q)$. Lemma 4.2 shows that $F_{p, q} Z\left(\Phi_{M}\right) F_{p, q}^{-1} \subset G L(n, \mathbb{C})$. Hence, by Lemma 4.5 we have $F_{p, q} Z\left(\Phi_{M}\right) F_{p, q}^{-1} \subset$ $U(p, q)$. So $Z\left(\Phi_{M}\right) \subset F_{p, q}^{-1} U(p, q) F_{p, q}$, which equals $W(p, q)$ by definition.

In order to prove the other inclusion, $W(p, q) \subset Z\left(\Phi_{M}\right)$, note that for every $t \in \mathbb{R}$

$$
\begin{aligned}
F_{p, q} \varphi_{M}(\exp (t)) F_{p, q}^{-1} & =F_{p, q}\left(\begin{array}{cc}
e^{2 \pi i m t} I_{p} & 0 \\
0 & e^{-2 \pi i m t} I_{q}
\end{array}\right) F_{p, q}^{-1} \\
& =e^{2 \pi i m t} I_{n},
\end{aligned}
$$

which commutes with everything in $G L(n, \mathbb{C})$. Let $B \in W(p, q)$. Then $F_{p, q} B F_{p, q}^{-1} \in$ $G L(n, \mathbb{C})$ by definition. So $F_{p, q} B F_{p, q}^{-1}$ commutes with every element of $F_{p, q} \Phi_{M} F_{p, q}^{-1}$, and hence $B$ commutes with every element of $\Phi_{M}$. Therefore $W(p, q) \subset Z\left(\Phi_{M}\right)$.

Proof of Theorem 4.7. Let the PM-blocks of $M$ be $M_{1}, \ldots, M_{r}$. By Proposition 3.6

$$
Z\left(\Phi_{M}\right)=Z\left(\Phi_{M_{1}}\right) \times_{\iota} \cdots \times_{\iota} Z\left(\Phi_{M_{r}}\right) .
$$

For each nonzero $M_{i}$, Proposition 4.8 shows that $Z\left(\Phi_{M_{i}}\right)=W\left(p_{i}, q_{i}\right)$. If all of the PM-blocks are nonzero, then

$$
Z\left(\Phi_{M}\right)=W\left(p_{1}, q_{1}\right) \times_{\iota} \cdots \times_{\iota} W\left(p_{r}, q_{r}\right) .
$$

By the definition of PM-block form, the only block that can be zero is the last one, $M_{r}$. Suppose that $M_{r}$ is zero. Then $\Phi_{M_{r}}=\left\{I_{2 n}\right\}$, so $Z\left(\Phi_{M_{r}}\right)=S p\left(2 n_{r}, \mathbb{R}\right)$, and hence

$$
Z\left(\Phi_{M}\right)=W\left(p_{1}, q_{1}\right) \times_{\iota} \cdots \times_{\iota} W\left(p_{r-1}, q_{r-1}\right) \times_{\iota} S p\left(2 n_{r}, \mathbb{R}\right) .
$$

\section{TORUS ACTIONS ON SYMPLECTIC ORBI-SPACES}

In this section, we characterize all symplectic orbi-spaces that admit an effective symplectic $T^{k}$ action. The main theorem is the following. It was proved in [W] for $n=2$ and $k=1$, and in LT for $k=n$.

Theorem 5.1. A symplectic orbi-space $\mathbb{R}^{2 n} / \Gamma$ admits an effective symplectic $T^{k}$ action if and only if $\Gamma$ is conjugate to a subgroup of $U\left(n_{1}\right) \times_{\iota} \ldots \times_{\iota} U\left(n_{k}\right)$, where the $n_{i}$ 's are nonzero, the sum of the $n_{i}$ 's is $n$. 
Proof. $(\Longrightarrow)$ Suppose $\mathbb{R}^{2 n} / \Gamma$ admits an effective symplectic action. Lemmas 3.1 and 3.3 show that $\Gamma$ is conjugate to the centralizer of a $k$-torus $\Phi_{M}$ in $\mathbb{T}^{n}$ for some matrix $M$ in PM-block form. Let $r$ be the number of PM-blocks in $M$, and for each $i$, let $n_{i}$ be the number of rows in the $i^{\text {th }}$ PM-block. The $n_{i}$ 's are nonzero (since PM-blocks are nonempty), the sum of the $n_{i}$ 's is $n$, and since the rank of $M$ is $k$, it follows that $r$ is at least $k$. By Theorem 4.7 (or Proposition 3.6), we have

$$
Z\left(\Phi_{M}\right) \subset S p\left(2 n_{1}, \mathbb{R}\right) \times_{\iota} \cdots \times_{\iota} S p\left(2 n_{r}, \mathbb{R}\right) .
$$

Since $\Gamma$ is finite, it must be conjugate to a subgroup of $U\left(n_{1}\right) \times_{\iota} \cdots \times_{\iota} U\left(n_{r}\right)$. By combining factors if $r>k$, we see that $\Gamma$ is conjugate to a subgroup of $U\left(n_{1}\right) \times_{\iota} \cdots$ $\cdot \times_{\iota} U\left(n_{k}+\cdots+n_{r}\right)$.

$(\Longleftarrow)$ Suppose $\Gamma$ is conjugate to a subgroup of $U\left(n_{1}\right) \times_{\iota} \cdots \times_{\iota} U\left(n_{k}\right)$, where the $n_{i}$ 's are nonzero and their sum is $n$. Let $M$ be the $n \times k$ matrix in PM-block form with $k$ PM-blocks, the $i^{\text {th }}$ of which is $\left(\begin{array}{c}\mathbf{e}_{i} \\ \vdots \\ \mathbf{e}_{i}\end{array}\right)$, with $n_{i}$ rows, where $\mathbf{e}_{i}$ is the $i^{\text {th }}$ standard basis vector of the row space. By Theorem 4.7,

$$
Z\left(\Phi_{M}\right)=U\left(n_{1}\right) \times_{\iota} \cdots \times_{\iota} U\left(n_{k}\right) .
$$

Hence $\Gamma$ is contained in some conjugate of $Z\left(\Phi_{M}\right)$. Since $M$ has rank $k$, it follows that $\Phi_{M}$ is a $k$-torus. Therefore, by Lemma 3.1 we see that $\mathbb{R}^{2 n} / \Gamma$ admits an effective $T^{k}$-action.

Remark 5.2. This theorem can actually be proven almost as easily using only the results from Section 3

Remark 5.3. This result does not necessarily imply that if $\Gamma \subset S p(2 n, \mathbb{R})$ is isomorphic to a finite subgroup of $U\left(n_{1}\right) \times \cdots \times U\left(n_{k}\right)$, then $\mathbb{R}^{2 n} / \Gamma$ admits an effective symplectic $T^{k}$ action; the $T^{k}$ action is only guaranteed for some representation of $\Gamma$ in $S p(2 n, \mathbb{R})$. We believe that the question of whether such an action exists for all representations of $\Gamma$ is open.

Corollary 5.4. All symplectic orbi-spaces admit a circle action.

Proof. Let $\mathbb{R}^{2 n} / \Gamma$ be a symplectic orbi-space. Since $\Gamma$ is a finite subgroup of $S p(2 n, \mathbb{R})$, and hence compact, it must be conjugate to a subgroup of $U(n)$.

Corollary 5.5 ([ $[\overline{\mathrm{LT}}]) . \mathbb{R}^{2 n} / \Gamma$ admits an effective symplectic $T^{n}$ action if and only if $\Gamma$ is conjugate to a subgroup of $\mathbb{T}^{n}$ (or equivalently, $\Gamma$ is contained in some torus).

Proof. In the statement of Theorem 5.1, since $k=n$, we must have each $n_{i}=1$. So $\Gamma$ is conjugate to a subgroup of $U(1) \times_{\iota} \cdots \times_{\iota} U(1)$, where there are $n$ copies of $U(1)$, which equals $\mathbb{T}^{n}$.

The preceding corollary allows us to easily find examples of orbi-spaces $\mathbb{R}^{2 n} / \Gamma$ that don't admit effective symplectic $n$-torus actions.

Example 5.6 ([STW]). Let $\Gamma$ be a non-abelian subgroup of $U(2)$, for example the representation of $D_{4}$ generated by $\left(\begin{array}{cc}i & 0 \\ 0 & i\end{array}\right)$ and $\left(\begin{array}{ll}0 & 1 \\ 1 & 0\end{array}\right)$. Since $\Gamma$ is not abelian, it is not conjugate to a subgroup of $T^{2}$, so $\mathbb{R}^{4} / \Gamma$ does not admit an effective symplectic $T^{2}$ action. 
Corollary 5.7. If $k>\frac{n}{2}$ and $\mathbb{R}^{2 n} / \Gamma$ admits an effective symplectic $T^{k}$ action, then $\Gamma$ is conjugate to a subgroup of $U(2 n-2 k) \times_{\iota} \mathbb{T}^{2 k-n}$.

(Note that we do not claim the converse.)

Proof. In the statement of Theorem 5.1 let $a$ be the number of values of $i$ such that $n_{i}=1$. There must be $(k-a)$ values of $i$ such that $n_{i} \geq 2$, so the sum of all of the $n_{i}$ 's, which must equal $n$, is at least $2(k-a)+a$. So $n \geq 2 k-a$, which implies $a \geq 2 k-n$. The product of $2 k-n$ factors of $U(1)$ is $\mathbb{T}^{2 k-n}$. The product of the remaining factors is a subgroup of $U(n-(2 k-n))=U(2 n-2 k)$.

\section{REFERENCES}

[BtD] T. Bröcker and T. tom Dieck, Representations of Compact Groups, Springer-Verlag, 1985.

[LT] E. Lerman and S. Tolman, Hamiltonian torus actions on symplectic orbifolds and toric varieties, Trans. Amer. Math. Soc. 349 (1997), 4201-4230. MR 98a:57043

[MR] J. E. Marsden and T. S. Ratiu, Introduction to Mechanics and Symmetry, 2nd ed., Springer-Verlag, 1999.

[MS] D. McDuff and D. Salamon, Introduction to Symplectic Topology, Oxford University Press, 1995. MR 97b:58062

[STW] S. F. Singer, J. Talvacchia and N. Watson, Nontoric Hamiltonian circle actions on four-dimensional symplectic orbifolds, Proc. Amer. Math. Soc. 127 (1999), 937-940. MR 99f: 57043

[W] N. Watson, Symplectic vector orbi-spaces with torus actions, Senior paper, Haverford College, 1997.

Département de Mathématiques, École Polytechnique Fédérale de Lausanne, CH1015 Lausanne, Switzerland

E-mail address: tanya.schmah@epfl.ch 